\title{
Genetically Modified Animals in Pharmaceutical and Food Chains
}

\author{
Ashok Kumar*, Satish Kumar Sharma \\ Department of Pharmacy, Glocal University, Saharanpur, Uttar Pradesh, India
}

Received October 21, 2020; Revised December 21, 2020; Accepted December 27, 2020

\section{Cite This Paper in the following Citation Styles}

(a): [1] Ashok Kumar, Satish Kumar Sharma , "Genetically Modified Animals in Pharmaceutical and Food Chains," Advances in Pharmacology and Pharmacy, Vol. 8, No. 4, pp. 72 - 80, 2020. DOI: 10.13189/app.2020.080403.

(b): Ashok Kumar, Satish Kumar Sharma (2020). Genetically Modified Animals in Pharmaceutical and Food Chains. Advances in Pharmacology and Pharmacy, 8(4), 72 - 80. DOI: 10.13189/app.2020.080403.

Copyright $\subseteq 2020$ by authors, all rights reserved. Authors agree that this article remains permanently open access under the terms of the Creative Commons Attribution License 4.0 International License

\begin{abstract}
The term "genetically modified organisms (GMO)" is becoming a contentious issue as its advantages both Potential biomedical impacts and side impact on the economy are bolstered for food suppliers and users. GM animal feed is an emergent method that may affect livestock as well as the pharmaceutical industry. Whilst two pharmaceuticals have yet to join the industry, food materials derived from GE animals. In order to revisit the progress in identifying the main causes, the study was carried out. Affecting GM applications adoption and their possible risks and advantages, GM-derived food products have still not entered the market. However, the active conversation on GM crops and the current conversations on a basis with socio-economic concerns involving the incorporation with GM animals through food and pharmaceutical chains lay the protection and values of food and medicinal products derived from the cloned animals. The same key influences driving the introduction of genetically modified animal products in agriculture including pharmaceutical chains were its endorsement by the population and farmers, government decisions, human wellbeing, animal welfare, impact on environment and conservation. Throughout this paper we explored the financial and leadership benefits and drawbacks of genetically modified animal or pharmaceutical products, and their effect on their introduction.
\end{abstract}

Keywords Agriculture, Food Chains, Genetically Modified Animals, Genetic Engineering, Pharmaceutical, Recombination

\section{Introduction}

Genetics is about customizing an organism's mutation by using its genetic information. Some genetic engineering uses this principle of hybridization. Recombination is indeed the process by that a new genes are implanted further into microorganisms "plasmid" DNA. The protein recognized as the restrictive enzyme should be cut off in the DNA. This constrictive enzyme must have a particular form that will allow the passage of DNA. A specific moment throughout the DNA series at which the DNA could be cut does seem to be the enzymatic limitation. When this limiting enzyme reductions, a "sticky side" appears to be left that helps attach the novel gene during that point. This same new group of DNA, named the "DNA ligase," is connected to this different enzyme, which is genetically engineered and many updated models with bacteria were also established with either the gene encoding. Genetic modifications may be made for plants as well as animals[1].

The use of in vitro nucleic acid therapies (for instance rDNA), as a result of GMOs, including cell fusion, contributes to overcoming the multiple metabolic reproduction or recombinant obstacles. techniques. In many other phrases, they result from the transformation in biological profile to accomplish desirable properties other than conventional breeding and selective practices. Understanding It is important to secure the advantages of biotechnology as well as the way humans and also the world from severe GMO adverse reactions [2]. 
Species is genetically changed by the insertion, removal or deletion of such DNA series in a way that is not normal to a species. It involves modifying an animal's unique characteristics or add a new trait, such as resistance to disease or increased growth. DNA is an organism's genetic material and carries directions for all of the features inherited by an organism. Therefore, property acquired in the genetic makeup of an animal can be passed on to the next generation[3].

Although this technology has been used to date to generate enzymes in plants for livestock and micro-organisms, the possible application of genetic modification techniques to animals is also being investigated. A few international organizations have already issued recommendations for the health evaluation of these animals and their related products, along with the FAO / WHO and the US Food and Drug Administration[4].

Genetically modified (GM) animal production is an important approach which could possibly Effect on animal and pharmaceutical industry. Various genetically modified mice, as well as rabbit including pigs are created to be used in personal or university laboratories to research the operation and function of genetic engineering. In addition to these GM products that were designed for therapeutic purposes, for example, Goldfish ${ }^{\circledR}$ will be the primary GM species to be sold, a GM Zebra fish through such a fluorescent genes to glow underneath UV light into the night. for development, disease resistance or product quality (milk, meat etc.)[5]. Other applications have been formed, like EnviropigTM, to minimize the environmental footprint of farming (- for example, Lastly, genetic modification should be utilized for the creation of milk \& white eggs, or any liquid of the sort (e.g. blood), individual antibodies, animal products, as well as for development of medicinal proteins, like GM animals (goat's, cows, dogs, chickens, pigs, or rabbits). or human transplant or xenotransplantation products.

Generation of pharmaceutical human proteins in the transgenic animals is indeed a small undertaking, involving only a few firms. No product has yet reached the market after ten years of research but the stewards of this emerging technologies hope to accomplish this in the next few years. Most research is currently aimed at products for the industrialized nations[6].

A transgenic animal is that by definition an animal whose genetic material has been modified by methodologies other than those used in conventional breeding that included selected genes from other animals or species. In many other words, an animal changed by having recombinant DNA introduced via human intervention. A mouse, generated in 1981, was the very first transgenic animal to hold a gene that created the animal impervious to cancer. A sheep formed in 1985, was the first transgenic farm-animal. Biotechnology companies and research institutions involved in the development and production of pharmaceuticals use transgenic animals for 3 distinct ends[4].

- As an investigation model. An example is the modification of an animal's genetic makeup in such a way that it develops a disease that is close to that of humans. Hence the animal is often used as a guide for seeing how a disease progresses and responds to medications.

- As an assay kit. Genetically modified animals can be used to make a human protein. When this protein is tried to apply to the transgenic animal generated by an external entity, the animal's immune response is an obvious sign of the protein pureness to be tested for[3].

- As pharmaceutical processing unit. The genes which code for the production of a human protein are inserted into an animal's genome, which then in turn generates the human protein, mostly through transgenic technology like micro-injection.

This particular phase of genetically modified animal production that has emerged recently has a new name: pharming. Pharming is manufacturing of human pharmaceutical proteins in transgenic farm animals. Altering the composition of milk is the principal strategy in most pharming cases. Cow mammary glands generate large quantities of milk, a solution rich in proteins that can be obtained non-invasively[7].

Although economic analyzes of the potential risks and assistances of GM harvests are broadly labelled and utilized, in the animal and pharmaceutical chains there is very little analysis of GM applications. It is because, for a number of reasons, animal genetic engineering has progressed much slower than the crops, like financial, technological, human wellbeing, the climate and the conservation of animals considerations. This article looks at the driving forces impacting transgenic animal adoption and implementation from a socio-economic and the life science perspective[8].

In a much more broad way, Improvements in agricultural biotechnology were expected to exist in economic terms for farm staff, producers and consumers (including, not restricted to genetic modifications in, animals). The evolution of GM types of fish somewhat higher than anti GM fish, for instance, is projected to reduce farming values, e.g. feed costs, though throughout the form of lower prices, as buyers gain economic benefits. The distribution of these benefits depends, however, on different factors, like technology (cost reduction or quality enhancement applications), regulatory framework and competitive spirit (concentration ratio, market power of suppliers, etc.), transparency of information (labeling and traceability programs, etc.), relative prices, consumer acceptance etc. Other external costs should also be regarded in the actual financial evaluation aside from the direct economic effects. Transgenic animals in specific can provide significant benefits for Consumers throughout the context of nutritious food from healthy animals, 
enhanced goods such as food with various health benefits as well as the safer world in a broader context by reducing animals farming's environmental impacts. Through order to prevent unintended environmental harm or increased threats to humans and even animal welfare, the introduction with animal biotechnology, however, has been closely controlled and wellbeing[1].

There are several years of work being carried out a modern GMO may be regarded for common use in labs afterwards. The above-mentioned risks should be checked and the potential effects again for food web analyzed. New GMO maize, for instance, can join the food web or be eaten by animals, so the effect with GMO on agricultural products must be investigated entering the food chain[8].

Human health and ecological risks associated with GMOs are indeed the core goals of their future usage assessment. Allergy (reaction to such proteins), possible toxicity and combined consequences of further than one feature of even an organism were analyzed as far as human well-being is concerned. From an environmental view, potential impacts for mutation-flow effects (through organism to organism) but production of tolerance (e.g. tolerance to pesticides) on the organisms without goal are analyzed.[3], [4].

In the private sector, most of the intermediate goods and methodologies which allow the advancement of GMOs, such as molecular fingerprinting and transformation technologies, are presently under protection of intellectual property rights. Public sector scientists therefore have less chance of gaining access to such products and methodologies, particularly in developing countries. This restricts their ability to develop enhanced crop or animal strains, such as GMOs that might help alleviate their specific local or national production problems. Thus the present crisis tends to bridge the gap between wealthier societies and poorer ones shown in table $1[1]$.

Table 1. A Selection of Genetically modified organisms that are Available Today

\begin{tabular}{|c|c|c|}
\hline GMO & $\begin{array}{c}\text { Genetic } \\
\text { modification }\end{array}$ & $\begin{array}{c}\text { Persistence of genetic } \\
\text { modification }\end{array}$ \\
\hline Maize & Insect resistance & Decreased insect damage \\
\hline Soybean & Herbicide tolerance & Better weed control \\
\hline Cotton & Insect resistance & Decreased insect damage \\
\hline $\begin{array}{l}\text { Escherichia } \\
\text { coli K } 12\end{array}$ & $\begin{array}{c}\text { Manufacture of } \\
\text { chymosin or rennin }\end{array}$ & Employed in cheese-making \\
\hline Carnations & Change of colour & $\begin{array}{c}\text { Production of diverse flower } \\
\text { varieties }\end{array}$ \\
\hline
\end{tabular}

\section{Material and Methods}

Exploratory Studies seek to rapidly trace the key principles behind a science field and the major sources including forms of evidence accessible. Scoping research guidelines were developed to provide the recommendations on how to recognize the socio-economic dimensions of related documents (keywords, journal articles, web sources, etc.) In these guidelines, strict constraints on the use of two specifically classify existing research and to analyses compilation and reporting processes at the outset, key terms were prevented. The technique isn't really linear but iterotic, enabling scientists to engage and stage by reflecting and taking actions, where appropriate, to make a stable literature scope.[9].

In the review of the findings several publications were considered: electronic databases, systematic reviews, key journal articles and agency contract. The research method, the keyword and reading comprehension research questions as well as interpretations were developed for electronic information internet, CD-ROM, etc.). The sources lists as well as bibliographies of study identified through database searches were to be reviewed to ensure that they were utilized in the scope exercise. A further crucial element was to search key journals by hand; this helped identify missed studies in searches of databases and reference lists. Finally existing expertise and networks would be able to produce research information. The analysis was thus improved by contacting relevant Field-based national or regional organizations, EU initiatives and/or EU study funding assist to manual library resources and to classify unpublished work. Even so, related journals in several other languages (e.g. French, Spanish, Dutch and so on.) have been included along with studies given that the significant topics shown in the article (subject, method used, major results etc.) is accessible inside an abstract throughout English (or even the major information used for the graphic) or the principal information has been transferred in English for diagrammatic purposes.[2].

For the compilation and standardization of all useful details on document a database charting method has been established. The type contained general information (e.g. purpose, source, etc.) from each analysis and detailed information (e.g. problems of regulation, genetic engineering, design methods, main conclusions, determining factors in applying GM technology, localization, impact steps, sources of evidence, secondary results, etc.). Thus, throughout the form of tables or even a chart, the main features and analysis were seen. The findings formed the framework of the research. A sum of 150 reports have been obtained from different sources.[7], [9].

\section{Socio-Economic Factors Influencing Genetically Modified Animal's Introduction}

A third of the papers studied concerned food webs and that only a relatively limited percentage of pharmaceutical 
companies (30\%) and approximately half of study comprised transgenic analyses and around one quarter of analytical or econometric analysis. This conveys a sense of potential feedback GM animal applications, instead of actual inflation statistics. Between 2002 and 2005 (40 per cent) and in more recent years (35 per cent after 2010), many studies were published[10].

The analysis of approaches for the assessment of the economies of GM animals indicates that GM implementations are, in general, highly limited, while there is considerable potential for increasing the productivity of animal supply chains including pharmaceutical goods. Empirical research into economic considerations including the value and progress of incorporating GM technologies in animal including medicinal products is significant in contrast with genetically modified crops. For animal GM applications, most economic analyses focus on GM applications pertaining to the implementation of GM hormones or GM vaccines in animals. There is to a large extent a lack of economic study of genetically engineered products of organisms alone (e.g. germline adoption with foreign DNA). In comparison, several other experiments are at the field or lab stage not even at the top of the hierarchy. A broad variety of approaches and procedures are used to evaluate the external advantages and disadvantages of GM applications such as simulation scenario analysis, quantitative economic models, econometric analysis and telephone qualitative interviews[11].

The key issues impacting the (future) use of genetically modified animal technologies in cattle and drugstores the literature studied range from the acceptance by the public and production companies to public policies. Other factors are also involved, like The following areas will be analyzed: environmental protection, health consequences, animal care and ethical issues [5].

\section{Public acceptance}

The general recognition of any transgendered animal production in food and pharmaceuticals is generally considered to be "condicio sine qua non." In determining the potential for animal biotechnologies globally, confusion regarding the response of customers is the main problem. The philosophical framework for technological adoption thus coincides with the consumer. The opinions of customers (hopeful vs unfavorable) are crucial for understanding GM adoptions and public expectations of GM technologies and their issues (health, food standards, unnaturally, ethics, the climate, animal protection and social welfare, etc.). Several studies focused on those issues[12].

Numerous researches show that, where food or animals are involved, public acceptance of GM application is the lowest. An investigation conducted in the U.S. has confirmed the reason that applications for plants were more sponsored than applications for animals. A global pool of the FAO estimates that 62 per cent of all respondents around the world opposed the use of biotechnology to improve the productivity of farm animals. Another situation would be that 68 percent of users do not agree through the assertion of producing transgenic fish, that increases processing quality, for example, for Pew Nutrition and Biotechnology.[1].

Another research indicates that end-user biotech acceptance differs considerably by field of application and world geography. Most commonly embrace medical and pharmaceutical biotechnology linked to GM animals, because of perceived financial gains. Public acceptance ranges In Japan, 83 percentile comparison with many other GM implementations in developed nations to 70 percent. This same poorest acceptability ranking for fishing biotechnology throughout all fields is seen. The poor tolerability for GM fish can lead to a wide variety of reasons, include environmental problems. Considering geographical disparities, consumers are more applicable to developed nations, which will also benefit from the use of increased food production. from the use of this technology[2], [7].

Another work carried out in the U.S has also documented the reality that more assistance was given by plant applications then animal implementations. Animals resistant to diseases were the most commonly used in animal products, although many who give fresh beef, human body parts and increased development were the least accepted by respondents. Some observational research investigated the approval of consumers for such GM products, for example by demonstrating greater conventional market expectations towards GM pork. In this case, GM pork's negative perception may be offset by quality improvements, increased animal health and welfare, lower environmental impact, less residues as well as a discount on costs. Increased livestock welfare would have the every positive effect on customer choices, while the best benefit is improved in the ecosystem. This indicates that customers will be more valuable for transgenic pork than traditional pork by a substantial monetary reward as per this study and the existence of varying benefits. The amount of monetary compensation depends on the application of GM, too[8].

\section{Producers' acceptance}

There may also be questions concerning the implementation of a modern technology, including customers. Unsurprised on where the system functions in the potential, anxiety regarding growing dependency on suppliers of supply, assumption of higher import costs, coexistence issues at the development stage and manufacturing process separation, confusion about the results and the probable acceptance of consumers are among the key concerns of producers listed in the 
literature. It is also evident that acceptance by the producer will rely on the expected benefits (decrease of feeding expenses, raise yields, etc.) as well as cost and revenue allocation through the chain. It is often claimed that at one stage in the supply chain the cost in deployment innovations exists, while the gains are seen at another stage.[2].

Methodologies for the animal transgenes is, like the science of microinjection (i.e. transfer of DNA to something like a pronuclear and embryos cytoplasm, primarily by direct microinjection), have been initially incredibly wasteful, but recent technological discoveries have overcome many of these technical problems. However, GM animal devices to farm production were indicated to be theoretically more difficult to create than clinical preparations, leading to difficulties in the selection of the right gene mutations, in specific in the field of developmentally based transgenes is. Furthermore, big animals like cows, while related projects like these, involve considerable investment throughout a prolonged period of time, were also known to always have lengthy reproductive cycles. In comparison with mammals avian species can be developed quickly and are highly suited to the growth of commercially valuable and bioavailable proteins to be used in pharmaceutical and industry purposes in egg white, consequently having short breeding cycles as well as an elevated cost of inventory of eggs. Comparably, technological aspects, such as greater egg production which can be exploited more quickly, and also financial reasons understand the high levels of studies focused on transgenic fish, because as fish processing industry is growing quickly[10].

It has been suggested in the specific case of aquaculture that a business that manufactures In addition to consumer skepticism, a new growth-enhanced salmon could also be discounted by the fisheries itself. Equipped local fish production companies might fear fresh transgenic fish competitors and also radical changes in the economic structure of the sector. Moreover, retailers that have greater market control and are more consumer-friendly than manufacturers' methods cannot be clever to buying transgenic fish, as well as can be at threat of being marginalized by their customers. However, companies can be frightened by protestors' anti-GMO movements that could adversely affect the company's brand public image[4].

\section{Policy implications}

Public strategies impact the earnings of reserved investment in R\&D by means Mechanisms covering direct govt, research money, academic ownership rights law, federal regulations, fiscal and monetary policies, social policies as well as other data on environmental regulations. Various documents have been produced that provide understanding into the management with transgenic animal protein. Food protection and risk efficiency reviews are known to be critical measures in approaching these emerging technology implementations. A recently conducted study on behalf of the National Food Protection Agency (NFSA) to identify the ecological risk analysis requirements for EU marketing of GM fish. It's also been argued that, since one country's decisions can affect the other, various attitudes to decision-making should be aligned as far as possible[13].

Copyright (i.e. copyright, trademark and marking) affects the cause of a company's investment in R\&D by improving the company's capacity to collect rentals and the subsequent product income. In specific for biotechnology as well as transgenic animals, it's a very difficult matter. Transgenic animals are being debated in patents not only on legislative terms, but also on philosophical and moral matters, such as the media. Many of the natural products (like special antibiotics, microorganisms, nutrition etc.) have been successfully licensed, giving innovators a right to reproduction. It is unclear whether a material that occurs naturally will be copyrighted, since it lacks innovation and innovative interventions. Even so, it's indeed patentable to enhance, purify or alter a result of nature in a manner that is commercially useful. Organic samples not existing in nature can be patented if they have been stripped in its natural environments and affirm the general standards in patentability with respect to innovation, inefficacy, value and adequacy of disclosure.[11].

Labeling and labelling strategies may be a reason for customers to make their decisions consciously and for manufacturers to discriminate between their goods. If the EU has approved and reasonably viable genetically modified animals and produced produce appropriately labelled in the EU, it is debated if the things manufactured from genetically modified livestock should be branded on any other sector. The US FDA also considering the issue of the marking of GM salmon, although this would result in a different amount equivalent to GM crop food. Labeling regulations will result in additional costs, including the traceability costs. The economic costs of tracking and identification of animals produced from bioteches and their materials, and most other expenses which may be required to comply with legal standards (e.g. physical processing of GM fish), should be taken into account. Compliance costs potentially decrease private viability of the system, but the public will take advantage of the lowest risks. The relation among costs and advantages of the regulatory thus decides the social economic utility of the law[2].

\section{Economic Aspects of Genetically Modified Animals}

An emerging method that is supposed to have a significant influence on cattle genetic development may be known as transgenic extraction. The species with the 
international diameter with DNA incorporated into their gene and any changes made to their genomic sequences were either transgenic or genetically modified (GM) species. Transgenic animals are being classified into three groups and have multiple possible applications[14]:

i). Data on human disorders and gene expression and regulation;

ii). Obtain high performance medical treatment materials (biopharmaceuticals and engrafting)

iii). Increasing food production for human food in both quantity and/or quality;

The priority is already on the last several 2 categories in this analysis. A research on the financial benefits and drawbacks of GM animals first from viewpoint of the supply chains was undertaken to determine the use of Traditional breeding.[3].

Scoping approaches are structured to chart the core theories underlying a research field and the core references and forms of evidence accessible. According to the meanings of the study criteria for the recognition of related articles (keywords, publications, online references, etc.), the key concepts are research issues (but how the political and financial implications of the implementation of genetically modified animals through food chains including drug system is or the techniques used), from 1996-2010 that quest approach was built based on research problems and keyword descriptions and core ideas for the digital databases (e.g. Twitter, CD-ROM, etc.). Including basic information by each analysis and even more detailed information (such as the type of gene editors, the economic consequences, regulatory issues, procedures, key findings, the factors influencing the use of GM techniques, the geographical location, therapy outcomes, the data s) every one of the related journal data was compiled and organized in such a data map format..)[7].

\section{GM animal economy in livestock feeding chains}

In general it depends on how biotechnical factors impact production prices, product quality or both transgenic organisms' economic impact on the sector. Biotechnologies, whether plants or livestock, can basically be categorized into two large economic classifications: a) reducing / enhancing prices, and b) increasing efficiency.[11].

Theoretically, cost reduction and increasing technologies will boost the income of the manufacturers by authorizing a certain quantity of a commodity to be generated at a lower price or a better production amount at the very same cost. Which would in the long run lead to a lower market burden in such a competitive setting. Of the food markets that will in turn favor consumers, while theoretically compensating the profit increase of the producers[8].

Applications for quality enhancement were developed primarily to improve milk composition, while other programs sought to enhance the quality of meat as well as other non-food characters. Milk production represents an attractive example of genetic modification. Bovine were generated that can over-express human lactoferrin (rhLF) in their recombinant milk. It is used in public health services for a wide range of potential applications such as prevention and management of infectious and parasitic diseases [3]. RhLF dairy is a functional food, used primarily in the pharmaceutical sector that offers new healthcare benefits such as improved disease prevention and improving gastrointestinal health and a greater adaptation to child consumption.

Improved quality food goods will theoretically raise farmers' benefits from expanding the supermarket. It is likely that improved quality food will, when the demand of the market improvements in conditions, be marketed for consumers at increased costs comparison to the normal food [9]. It creates fresh niches and therefore splits the sector and modifies the whole production chain. Therefore, regard to consumer distinction between elevated cost and lower quality goods, the income inequality is harder to determine.

To improve the quality of pork meat, IGF-1 transgene was found to have helped to reduce carcass metabolism and total the lean mass, which makes every hog worth an extra $\$ 6$ on the market. Pigs have also been developed to produce intracellular omega-3 fatty acids that are implicated in the prevention of cardiovascular problems. This is asserted to become a more economic, secure and energy stores for meat enrichment to address the increasing requirement of omega-3 fatty acids within the average food based on current practice of feeding fishmeal [13]. A major argument among those who favor and against omega-3 pork meals marketing was founded by the release of this research.

The latter has curiously claimed that "one can change an animal's genome so that people can continue eating their own unhealthy eating habits," i.e. eat junk food. Research authors reported that omega-3 pigs can decrease the risk of unexpected heart disease by 40 per cent, indicating that health improvement through food changes is easier than by changing the behaviors of the consumers[6].

Examples of cost reduction or quantity enhancement projects are programs aimed at increasing output, improving animal welfare through improved disease resistance, increasing carcass and milk production yields and through reproductive success. An approach to increasing the production of sow milk was achieved by altering the Milk ingredients, like lactose. It was approximated that the more proper and effective meat manufacturing, focuses on the development of healthful, rapidly growing piglets, will also lead to an extra $\$ 2.5$ per liter, a $10 \%$ increase in milk supply create an overall financial advantage of \$29 million / year in the U.S. pork 
industry, taking into account the typical hog price of $\$ 50$ /cwt[10], [13].

\section{GM animal economy in pharmaceutical chain}

Conditional human therapeutic protein production from blood or tissue extracts is a costly and inefficient labor process that carries the risk of being contaminated with human pathogens. The production, by recombinant bacteria or cell cultures, of human therapeutic protein has eased these difficulties and provided patients with several therapeutic proteins. These recombinant systems, however, have certain technical limitations and could only be generated at high manufacturing costs. Biopharming seems to be the production of drug molecules in animals and plants tissue farming systems, which is considered to be the next major advancement of both farming which pharmaceutical processing [4], [5]. For farmers, biopharming's appeal is to produce high-value, niche goods that shift conventional farming away from the production of commodities. Biopharmaceutical guarantees a role contributes for drug industry reduce production costs as compared to cell culture. The advantages of biopharming would be that lower cost drugs produced extra rapidly for the general public. Biopharmaceutical benefits compared with Methods for cell cultivation provide lower generation and processing costs, faster distribution, better spread, higher protein produces and increased protection.

A movement is ongoing to use polyclonal antigen therapy. The individual, unique epitope of the monoclonal antibodies is known as coniferous balloons while polyclonal treatment can be represented also as method of a gun. A value add of primary antibodies in hyper immunized animals against a chosen target is that the host usually chooses most immunogenic epitopes for them. Biopharmaceutical companies are also testing a range of new methods involving biochemical methods, immunotherapy and vaccination methods for a number of disorders such as Helps, autism and even allergies utilizing cytokine types, bacterial toxins including cellular components. The development of anti-cytokine therapy for treating HIV infects, for example, includes Neovacs SA (Paris, France). Colorectal cancer therapy also recently reported generation of recombinant polyclonal antibodies. In the future, polyclonal derived antibody therapy looks bright with this sort of progress[1], [2].

Though the biopharming approach seems feasible, many attempts at commercial exploitation have been halted by the needed financial involvement during the long process of growth. Several business entities have withdrawn from transgenic biopharmaceuticals for various reasons in recent years[10].

\section{Economics of GM fish in aquaculture}

There is a greater interest in the production of GM in aquaculture than in Major reason besides terrestrial animals: easier to harvest fish eggs, and much more rapidly growing meat market besides farmed fish. Improved nutritional processing, temperature tolerance \& resistance to freeze, better diseases and metabolism (e.g. to minimize the necessity of salmonid fish fish diets), sterilization of fish, and fish pharmacy characterize the widely produced and most economically valued GM-fish. Around 50 fish species underwent genetic modification with over 400 combinations of fish / trait[13]. The U.S. especially FDA is now taking into consideration if a GM Atlantic salmon must be approved for promotion, which grows quicker and takes less food to develop. The first transgenic animal can be produced GM salmon to have been authorized for human use. Others claim, however, that GM fish may have already been marketed; comprehensive trials of growth-enhancing GM tilapia have been recorded in Cuba; and similarly, growth-enhancing GM carp might've been produced in China again for market; information is sparse and contradictory both in instances (Maclean, Spanish).)[8].

Transgenic fish's economic impacts vary from good to bad. The economic impact of increasing GM fish could be enormous: this fish would have much stronger growth unlike non-GM fish. Researches have already shown that growth has accelerated, especially for salmonids which reach maximum addressable market in much less than half of cases needed. by the same species for non-transgenic fish.

Furthermore, it is expected that the feed conversion ratio (FCR) will be more effective, i.e. the body weight obtained per kilogram of feed ingested. This could be an economical advantage, given that the feed cost accounts for over $50 \%$ of total salmon families operating costs. It has been projected that GM growth-intensified salmon production unit costs would fall by $20 \%$ to $50 \%[7]$. This decrease in costs could cause the global output to increase and the resulting decrease in market prices. As noted by researcher, price cuts may promote the consumption of To low-income families, new GM as well as non-GM salmon are sensitive to poorer nutritional requirements, while receiving high marginal social benefits[12],[13]. Related findings for other aquatic improvements like enhanced microorganisms including resistance, and altered metabolism, may be foreseen, although less relevant.

However, that the possible environmental consequences on wild animals of GM fish disappeared has overshadowed the debate and hindered their acceptance until now. Biological and physical protection systems may resolve these environmental issues. Only cultivators who breed them in protection restricted systems must buy sterile (triploid) and single gender (female) GM fish [7] [10]. Yet constructing these buildings costs $40 \%$ higher, and running $60 \%$ more than cages. This might in any way could the attractiveness of these goods. 


\section{Conclusions}

The growth of transgenic animals has progressed much slower than genetic engineering of crops that possibly have a significant effect on living as well as pharmaceutical restraints. Enhancements in animal biotechnology are required to give farmers, processors and consumers economic benefits. Many external costs must be taken into consideration in the final appraisal, both pro and con. apart from the direct economic consequences.

Biopharming is an uncharted ground for the farm, medical and government regulatory agencies and others. Because of the high cost, transgenic animals like pork, caprine, sheep and cattle are expected to yield high profits so that economic investment can be made feasible. That is why the main and most promising implementation for animal transgenesis is presently Manufacture of prescription high demand drugs, with a retail value of several thousand euros. However, several tries at commercial use were avoided, but still only two products were developed during the longer production period have managed to reach the market at present.

In aquaculture the interest in GM growth in domestic species is higher. This has several reasons; increased costs with fish growth as well as the production rates for feed quality would lead to costs being lowered, and consequently lower retail prices, but also illustrated why it may have major financial implications for GM fish. The example of development-enhanced GM fish indicates that product advantages can be minimized and gross margins improved without maximum delivery for products thanks to increased productivity growth and nutrient processing rates. In the ultimate evaluation of the implementation with transgenic animals, around the same period the environmental and health risks must be taken into account in detail. Indeed, major greenhouse gas emissions associated with GM fish farming may entail physical containment techniques that could theoretically restrict the economic attractiveness of GM fish.

An study of these concerns in general and that of the 3 cases of explicitly increased salmon, milk beef, and humanized polyclonal anti-corps developed by rabbits-indicates that there is currently no generalization from the life science perspective of the potential advantages and drawbacks of genetically modified animals.

\section{REFERENCES}

[1] M. Kramkowska, T. Grzelak, and K. Czyzewska, "Benefits and risks associated with genetically modified food products," Annals of Agricultural and Environmental Medicine. 2013.

[2] C. Mora et al., "Factors affecting the adoption of genetically modified animals in the food and pharmaceutical chains,"
Bio-based Appl. Econ., 2012.

[3] L. J. Frewer et al., "Genetically modified animals from life-science, socio-economic and ethical perspectives: Examining issues in an EU policy context," New Biotechnology. 2013.

[4] P. Nambisan, "Recombinant DNA Technology and Genetically Modified Organisms," in An Introduction to Ethical, Safety and Intellectual Property Rights Issues in Biotechnology, 2017.

[5] J. C. Cheftel, "Emerging risks related to food technology," NATO Sci. Peace Secur. Ser. A Chem. Biol., 2011.

[6] M. J. Punt, T. J. Venus, and J. H. H. Wesseler, "Dairies investment decisions in voluntary GM-free labeling standards in Germany,” Int. Conf. Coexistence between Genet. Modif. non-GM based Agric. Supply Chain. (GMCC)>GMCC-15 Seventh GMCC, 2015

[7] A. S. Bawa and K. R. Anilakumar, "Genetically modified foods: Safety, risks and public concerns - A review,” Journal of Food Science and Technology. 2013.

[8] F. Forabosco, M. Löhmus, L. Rydhmer, and L. F. Sundström, "Genetically modified farm animals and fish in agriculture: A review,” Livestock Science. 2013

[9] T. L. of Congress, "Restrictions on Genetically Modified Organisms,” 2015, 2015.

[10] H. Azadi and K. Ammann, "How Do socio-political disputes concerning GMOs influence decision-making in developing countries?," in Genetically Modified Organisms in Developing Countries: Risk Analysis and Governance, 2017.

[11] 139-41. Retrieved fromhttp://www.ncbi.nlm.nih.gov/pubm ed/23427432 [Control of Aedes aegypti larvae (L) with Poecilia reticulata Peter, 1895: a community experience in Taguasco municipality, Sancti Spíritus, Cuba]. Revista Cubana de Medicina Tropical, 58(2) et al., "Development of a population suppression strain of the human malaria vector mosquito, Anopheles stephensi.,” Malar. J., 2013.

[12] D. J. Mandell et al., "Biocontainment of genetically modified organisms by synthetic protein design," Nature, 2015.

[13] J. Bovay and J. M. Alston, "GMO food labels in the United States: Economic implications of the new law," Food Policy, 2018.

[14] L. Kaestner, A. Scholz, and P. Lipp, "Conceptual and technical aspects of transfection and gene delivery," Bioorganic and Medicinal Chemistry Letters. 2015.

[15] Chandrashekara, Kn, and K. Srinivas. "New Analytical Method Development for the Active Pharmaceutical Ingredients Using Visible Spectrophotometry." International Journal of General Medicine and Pharmacy (IJGMP) 2.2 (2013):55-64

[16] Ara, Talat, and Ak Choudhary. "An Attempt to Propagate and Conserve Withania Somnifera (L). Dunal: A Polypharmaceutical Plant Through In Vitro Culture." Impact: International Journal of Research in Applied, Natural and Social Sciences (IMPACT: IJRANSS) 2.4 (2014):159-164

[17] Goswami, Joydeep. "Employment of Workers and Capital 
by Size of Firms-A Study of Indian Pharmaceutical Industry." Impact: International Journal of Research in Business (Management IMPACT: IJRBM) 8.2 (2020):1322

[18] Majumdar, Dibyendu Dutta. "Recent updates on pharmaceutical potential of plant protease inhibitors." International Journal of Medicine and Pharmaceutical Science (IJMPS) 3.4 (2013): 101-120.
[19] Shah, Mehul K., Chetan B. Bhatt, and Jaimin B. Dave. "Nir spectroscopy: Technology ready for food industries applications." International Journal of Applied and Natural Sciences (IJANS) 5.1 (2016): 129-138.

[20] Bhutia, Karma L., and Wricha Tyagi. "Use of Sequence Specific Nucleases for Site Specific Modification of Plant Genome For Crop Improvement." International Journal of Agricultural Science and Research (IJASR) 7.5 (2017):491-502 